OPEN ACCESS

University of Dundee

\title{
Prediction of Pathological Complete Response to Neoadjuvant Chemotherapy for Primary Breast Cancer Comparing Interim Ultrasound, Shear Wave Elastography and MRI
}

Evans, Andrew; Whelehan, Patsy; Thompson, Alastair; Purdie, Colin; Jordan, Lee; Macaskill, Jane

Published in:

European Journal of Ultrasound

DOI:

10.1055/s-0043-111589

Publication date:

2018

Document Version

Peer reviewed version

Link to publication in Discovery Research Portal

Citation for published version (APA):

Evans, A., Whelehan, P., Thompson, A., Purdie, C., Jordan, L., Macaskill, J., Waugh, S., Fuller-Pace, F., Brauer, K., \& Vinnicombe, S. (2018). Prediction of Pathological Complete Response to Neoadjuvant Chemotherapy for Primary Breast Cancer Comparing Interim Ultrasound, Shear Wave Elastography and MRI. European Journal of Ultrasound, 39(04), 422-431. https://doi.org/10.1055/s-0043-111589

\section{General rights}

Copyright and moral rights for the publications made accessible in Discovery Research Portal are retained by the authors and/or other copyright owners and it is a condition of accessing publications that users recognise and abide by the legal requirements associated with these rights.

- Users may download and print one copy of any publication from Discovery Research Portal for the purpose of private study or research.

- You may not further distribute the material or use it for any profit-making activity or commercial gain.

- You may freely distribute the URL identifying the publication in the public portal.

Take down policy

If you believe that this document breaches copyright please contact us providing details, and we will remove access to the work immediately and investigate your claim. 
Prediction of Pathological complete response to Neoadjuvant chemotherapy for primary breast cancer comparing interim ultrasound, shear wave elastography and MRI

Advances in Knowledge

SWE stiffness after 3 cycles of NACT is strongly associated with pCR after 6 cycles.

Use of a 50kPa cut-off is more useful than using the percentage reduction in stiffness.

(c) Georg Thieme Verlag KG Stuttgart · New York.

Final published version available at https:/www.thieme-connect.de/products/ejournals/abstract/10.1055/s-0043-111589 


\section{Abstract}

Background: Prediction of Pathological complete response $(p C R)$ of primary breast cancer to NACT may influence planned surgical approaches in the breast and axilla. The aim of this project is to assess the value of interim shearwave elastography (SWE), ultrasound (US) and MRI after 3 cycles in predicting pCR.

Methods: 64 patients receiving NACT had baseline and interim US, SWE and MRI examinations. Mean stiffness at SWE, US and MRI diameter were measured at both time points. We compared four parameters with pCR status: a). Interim mean stiffness $\leq$ or $>50 \mathrm{kPa} ; \mathrm{b})$. Percentage stiffness reduction; c). Percentage US diameter reduction and d). Interim MRI response using RECIST criteria. The Chi square test was used to assess significance.

Results: Interim stiffness of $\leq$ or $>50 \mathrm{kPa}$ gave the best prediction of $\mathrm{pCR}$ with $\mathrm{pCR}$ was seen in 10 of $14(71 \%)$ women who had an interim stiffness of $\leq 50 \mathrm{kPa}$, compared to 7 of $50(14 \%)$ of women who had an interim stiffness of $>50 \mathrm{kPa},(p<0.0001)$ (sensitivity $59 \%$, specificity $91 \%$, positive predictive value $71 \%$, negative predictive value $86 \%$ and diagnostic accuracy $83 \%$ ). Percentage reduction in stiffness was the next best parameter (sensitivity $59 \%$ specificity $85 \%, p<0.0004$ ) followed by reduction in MRI diameter of $>30 \%$ ( sensitivity $50 \%$ and specificity $79 \%, p=0.03$ ) and $\%$ reduction in US diameter (sensitivity $47 \%$, specificity $81 \%, p=0.03)$. Similar results were obtained from ROC analysis.

Conclusion: SWE stiffness after 3 cycles of NACT is strongly associated with pCR after 6 cycles.

\section{Introduction}

Pathological complete response ( $\mathrm{PCR}$ ) is increasingly common after neoadjuvant chemotherapy (NACT) for primary invasive breast cancer. Early prediction of pCR may influence planned surgical approaches in the both the breast and axilla. Knowledge of response before the end of chemotherapy is helpful when 
planning complex autologous breast reconstructions especially if pre-reconstruction sentinel node biopsy is contemplated.

Interim assessment of response is usually carried out using MRI which has been shown to be superior to mammography and clinical examination in this clinical setting (1). In many series MRI is also superior to ultrasound (US) (1,2). Standard MRI assessment uses RECIST criteria which is based on changes in unidimensional measurements. However, both volumetric, functional and texture based MRI assessment have been shown to be superior at interim prediction, particularly in luminal and hybrid subgroups (3-6). MRI has the disadvantages of being expensive, time consuming and in many centres there are availability issues.

Shearwave elastography (SWE) is an ultrasound imaging method which allows reproducible quantification of lesional and peri-lesional stiffness and it has been shown to aid benign/malignant differentiation of breast masses $(7,8)$. It has also been shown to be an independent predictor of nodal metastasis and response to NACT at baseline $(9,10)$. SWE is fast to perform and interpret.

One previous study has assessed the value of SWE in predicting PCR at the end of NACT (11). The authors found that SWE performed similarly to MRI and better than US. Two previous studies have looked at interm assessment with SWE. On assessed interim prediction with SWE and strain elastography to response (12) while the other compared interim SWE alone to response (13) Neither of these studies compared the SWE findings with those of MRI or grey scale US, the modalities routinely used in this clinical setting. We therefore hypothesised that SWE may be useful in interim assessment of response in women undergoing NACT. The aim of this project was to assess the value of interim SWE compared to US and MRI after 3 cycles in predicting pCR after 6 cycles of NACT. 


\section{Materials and methods}

Sixty four patients with breast cancer receiving NACT were recruited into an ethically approved study which included baseline and interim US, SWE and MRI examinations. No patients were excluded after initial inclusion. Interim imaging examinations were performed after 3 cycles of chemotherapy, with patients proceeding to 6 cycles in total. All US scans were performed by one of five breast radiologists or one advanced radiography practitioner trained to perform and interpret breast ultrasound using an Aixplorer ${ }^{\circledR}$ ultrasound system (SuperSonic Imagine, Aix en Provence, France). These practitioners had between 7 and 22 years of breast ultrasound experience and had at least 12 months experience of performing SWE of solid breast lesions.

Four SWE images in two orthogonal planes were obtained. The ROI utilized in all cases was $2 \mathrm{~mm}$ in diameter. Maximum diameter was obtained from the US images. Mean stiffness in kPa was taken as the average of the values taken from four SWE images taken in two orthogonal planes. Imaging data was acquired prospectively, so without knowledge of the final pathological outcome. If the lesion was not seen on US then the US diameter was taken as $1 \mathrm{~mm}$ while SWE readings in the region of the previous abnormality were taken, this was identified by looking at the body mark on the previous US and viewing the pre-treatment MRI. Percentage reductions in tumour diameter and mean stiffness were calculated. Cut off values for percentage reduction in diameter and stiffness were chosen to give the same proportion of patients in the good response group as patients had a pCR at surgery. The percentage reduction in stiffness and US diameter were combined and assessed as a potential parameter. This was done by comparing the median values for US diameter and stiffness at baseline for the whole data set. The median values for US diameter values in $\mathrm{mm}$ were 4.8 times smaller than the stiffness values in $\mathrm{kPa}$. Therefore the US diameter values were multiplied by 4.8 and then added to the stiffness measurement to give a combination value. An absolute cut off value for interim SWE values of 
$50 \mathrm{kPa}$ was also applied as this is a cut off value established in the literature for the differentiation of benign and malignant masses $(7,14)$.

All MR examinations were performed on a 32-channel 3.0 Tesla (T) Siemens Magnetom Trio scanner (Erlangen, Germany) with a 7-channel open breast biopsy coil. Patients were imaged in a head-first prone position. Early post contrast T1 weighted sequences were used to obtain measurements of tumour on pre NACT and interim scans and RECIST criteria used to assign patient to assessment categories.

pCR was classified as an absence of any invasive cancer cells in the tumour bed at surgical resection after 6 cycles of NACT and an absence of nodal metastases at axillary surgery. However all women with a pCR in this study also had no DCIS in the tumour bed at resection.

Sensitivity, specificity and diagnostic accuracy were calculated for each modality and modality combination, and the chi-square test was used to assess the statistical significance of differences.

Means and associated standard deviations were calculated for lesion sizes and stiffness before treatment and at interim. Receiver operator characteristic (ROC) curves were produced and the area under the curve (AUC) determined using Medcalc software. A pairwise statistical comparison of the ROC curves was also performed. 


\section{Results}

Sixty four patients constituted the study group. The mean age was 52yrs (range 25-79yrs). Twenty-five patients had triple negative tumours, 21 had HER2 +ve cancers and 18 had luminal cancers. The first 3 cycles of chemotherapy in all patients consisted of 5-fluorouracil, epirubicin and cyclophosphamide (FEC). The mean pre NACT stiffness was $127.4 \mathrm{kPa}$ (range 55-289, SD =54) and the mean pre-treatment US size was $27 \mathrm{~mm}$ (range $8-45$, SD 9). pCR occurred in 17 of 64 (27\%) women (Figure 1) while 47 patients had residual disease (Figure 2). At interim scanning a residual US visible mass was seen in 62 of 64 (97\%) women. Of the 2 women with no US mass on interim scanning, one had a focal area of residual stiffness. Both of these patients had a pCR. The mean interim stiffness was $90.3 \mathrm{kPa}$ (range 17218, SD 55) and the mean interim US size was $21 \mathrm{~mm}$ (range 1-43, SD 10).

pCR was seen in 10 of $14(71 \%)$ women where masses had an interim stiffness value of $<50 \mathrm{kPa}$, compared to 7 of $50(14 \%)$ of women whose masses had an interim stiffness value of $\geq 50 \mathrm{kPa}, \mathrm{p}<0.0001$. Using this $50 \mathrm{kPa}$ cut off gave a sensitivity of $59 \%$, specificity $91 \%$, PPV $71 \%$, NPV $86 \%$ and a diagnostic accuracy of $83 \%$ (Table 1). Increasing or lowering this threshold did not improve overall performance.

The cut off value for percentage reduction in stiffness giving the same proportion of patients (17/64, $27 \%$ ) as those showing a pCR was $50 \%$. The cut off value for percentage reduction in US dimeter giving the same proportion of patients $(17 / 64,27 \%)$ as those showing a pCR was $61 \%$. The cut off value for percentage reduction in stiffness and US diameter combined and giving the same proportion of patients (27\%) as those showing a pathological pCR was $114 \%$. The associations between pCR and changes in US and SWE parameters based on these cut-off values at interim scanning are shown in Table 1 . The diagnostic accuracy using percentage reduction in stiffness, percentage reduction in US diameter and a combination of percentage reduction of stiffness and US diameter were $78 \%, 72 \%$ and $75 \%$ respectively. The percentage reduction in stiffness and percentage reduction in US diameter gave statistically 
significant results ( $p<0.0004$ and $p=0.03$ respectively). The combination of percentage reduction in stiffness and US diameter did not improve performance compared to that achieved by percentage reduction in stiffness alone (Table 1 ).

Baseline and interim MRIs were available for 59 of 64 (92\%) patients. Using a >30\% reduction in diameter as an indicator of response, the sensitivity, specificity and diagnostic accuracy of MRI were $50 \%, 79 \%$ and $71 \%$ respectively. The percentage reduction in MRI diameter and its association with pCR was statistical significant $(p=0.03)$ (Table 1$)$.

ROC analysis gave the following AUC measurements for predicting pathological complete response at interim assessment; change in MRI diameter 0.68 , percentage change in stiffness 0.82 , stiffness value at interim 0.78 , percentage change in US diameter 0.67 and percentage change in a combination of change in US diameter and reduction in stiffness 0.83 . The ROC curves are shown in Figures 3 to 7 . All these ROC curves shown on one graph are shown in Figure 8. The only statistically significant difference in AOC measurements following a pairwise comparison was that between percentage change in US diameter and the combination of US diameter change and stiffness change $(p=0.02)$.

Images where different imaging modalities gave differing interim estimates of response are shown in Figures 9 and 10 . 


\section{Discussion}

We have shown that interim SWE after 3 cycles of NACT using a simple threshold of $50 \mathrm{kPa}$ for mean elasticity is strongly associated with the chance of a pCR after 6 cycles of NACT. This simple threshold assessment has a stronger association with $\mathrm{pCR}$ than estimating the percentage reduction in stiffness. Both these parameters appear to outperform greyscale US assessment using uni-dimensional diameter and MRI using RECIST criteria when analysis is performed using cut off values. Analysis using ROC curves suggests that percentage change in stiffness and percentage change of a combination of US diameter change and stiffness change are the best parameters at interim scanning to predict $\mathrm{pCR}$ at the end of treatment. However assessed, the best parameters include SWE assessment.

A common problem using greyscale US to assess tumour response to NACT is that many cancers when treated go from an ovoid shape to a flatter, plaque like appearance. When this happens, the unidimensional diameter can remain unchanged even if the tumour volume has reduced considerably. This problem can be overcome by using volumetric assessments using a 3D probe. One study has shown the use of a 3D probe to be helpful in this clinical setting (15). However 3D probes only have a footprint of $4 \mathrm{~cm}$ so they are not able to measure the volume of large tumours. Many tumours also cause considerable posterior shadowing which can obscure the posterior border of the tumour and so interfere with volumetric assessment. Another problem when using US is that even when a pathological complete response has been achieved a residual mass of fibrous tissue is seen, measured and assumed to be viable tumour. One advantage of using SWE is that these residual fibrous masses with no residual cancer tend to be soft on SWE so assessment on SWE is less prone to errors of this nature. SWE benefits from being easy to perform and that the results have good reproducibility with intra-class coefficient (ICCC) for quantitative measurements between scans taken by different observers being 0.85 which is in the "near perfect" range if 4 images are taken in 2 planes. Stiffness measurements taken by different observers from the same scans have an ICCC of 0.99. Qualitaitve ssessments of SWE images 
(not performed in this study) are less reproducible $(7,14,16)$. Performance of SWE on breast masses have a few limitations. Lesions very deep in the breast can be difficult to assess and patients with breathing difficulties can also be impossible to scan accurately.

It is becoming more common to see pCR in breast cancer as NACT regimens become more effective, resulting in more women undergoing surgical resection of breast tissue even though no viable tumour is present (17). In response to this, clinical study of the use of percutaneous sampling of the tumour bed using vacuum assisted biopsy devices becomes more attractive (18). Before this can happen, imaging prediction of $\mathrm{pCR}$ has to improve. It is becoming clear that pure anatomical assessment of the tumour bed is not fit for purpose and that functional assessment either using MRI or other modalities such as PET/CT or SWE offer superior prediction $(3,5,19)$. It is also becoming clear that different immunophenotypes of breast cancer have different rates and patterns of response to NACT and that this necessitates a tailored approach by the radiologist when assessing response to NACT (4).

Response to NACT of the primary tumour is also important in guiding axillary surgery, as response in the axilla is closely correlated to response of metastatic disease in axillary lymph nodes. In patients who have biopsy proven axillary disease but appear to have a pCR prior to surgery there is now some evidence to suggest the safety of a sentinel node biopsy rather than an axillary clearance, albeit with a higher false negative rate than previously accepted (20). Thus correct and accurate assessment of response to NACT prior to surgery will influence both surgical options for the tumour bed and the axilla.

This study has a number of limitations. It comes from a single centre with a special research interest in SWE but as the technique is straightforward to perform we do not foresee difficulty in other centres replicating these results. The number of patients in the study is modest so differences in performance according to immunophenotype have not been assessed. It is possible that SWE may perform better in 
certain immunophenotypes in this setting as this is certainly the case when using SWE to predict response at baseline and when using MRI to predict $p C R$ at interim scanning $(4,21)$.

Two previous similarly sized recent studies have addressed the topic of assessing interim response of breast cancer to NACT using SWE. Their finding are comparable to our study. What is new in the current study is showing that SWE interim assessment is at least as accurate as grey scale US or MRI using RECIST criteria. No studies have been performed assessing the value of SWE in assessing response after 1 or 2 cycles of NACT. It is therefore unclear how soon SWE could be used to assess response after the commencement of NACT. A previous study has assessed the value of assessing response to NACT with SWE at the end of NACT. They found that a threshold of $30 \mathrm{kPa}$ was useful in predicting response. They found this cut off value more predictive than other higher values. They did not attempt to look at the value of percentage reduction in stiffness compared to baseline values (11).

In conclusion, SWE shows promise as a method of interim prediction of response in women with breast cancer treated with NACT and could be used to inform surgical decision making.

\section{Acknowledgements}

This work was funded by Breast Cancer Now 20120N46

\section{Figure legends}

Figure 1. Images showing baseline and interim US $(a, b), \operatorname{SWE}(c, d)$ and MRI $(e, f)$ in a woman who achieved a pCR correctly predicted by all modalities

Figure 2. Images showing baseline and interim US (a,b), SWE (c,d) and MRI (e,f) in a woman who had residual invasive cancer correctly predicted by all modalities

Figure 3. ROC curve demonstrating the relationship between change in MRI diameter between baseline and interim scanning and pCR. The AOC is 0.68 (95\% Cl's 0.54 to 0.79$)$

Figure 4. ROC curve demonstrating the relationship between percentage change in stiffness between baseline and interim scanning and pCR. The AOC is 0.82 ( $95 \% \mathrm{Cl}^{\prime} \mathrm{s} 0.70$ to 0.90 ) 
Figure 5. ROC curve demonstrating the relationship between stiffness at interim scanning and $\mathrm{pCR}$. The $\mathrm{AOC}$ is 0.77 (95\% Cl's 0.65 to 0.87 )

Figure 6. ROC curve demonstrating the relationship between percentage change in US diameter between baseline and interim scanning and PCR. The AOC is 0.67 (95\% Cl's 0.54 to 0.78 )

Figure 7. ROC curve demonstrating the relationship between percentage change in the combination of US diameter and stiffness between baseline and interim scanning and pCR. The AOC is $0.83\left(95 \% \mathrm{Cl}^{\prime} \mathrm{s}\right.$ 0.71 to 0.91 )

Figure 8. ROC curve demonstrating the relationship between the different parameters and pCR. 


\begin{tabular}{|c|c|c|c|c|c|c|}
\hline & pCR (n) & No $p C R(n)$ & $\mathrm{p}$-value & sensitivity & specificity & $\begin{array}{l}\text { Diagnostic } \\
\text { accuracy }\end{array}$ \\
\hline $\begin{array}{l}\text { Shearwave } \\
<50 \mathrm{kPa} \text { at } \\
\text { interim }\end{array}$ & 10 & 4 & $<0.0002$ & $59 \%$ & $91 \%$ & $83 \%$ \\
\hline $\begin{array}{l}\text { Shearwave } \\
\geq 50 \mathrm{kPa} \text { at } \\
\text { interim }\end{array}$ & 7 & 43 & & & & \\
\hline $\begin{array}{l}\text { Stiffness } \\
<52 \% \text { of } \\
\text { baseline }\end{array}$ & 10 & 6 & $<0.0004$ & $59 \%$ & $85 \%$ & $78 \%$ \\
\hline $\begin{array}{l}\text { Stiffness } \\
\geq 52 \% \text { of } \\
\text { baseline }\end{array}$ & 6 & 36 & & & & \\
\hline $\begin{array}{l}\text { US diameter } \\
<61 \% \text { of } \\
\text { baseline }\end{array}$ & 8 & 9 & 0.026 & $47 \%$ & $81 \%$ & $72 \%$ \\
\hline $\begin{array}{l}\text { US diameter } \\
\geq 61 \% \text { of } \\
\text { baseline }\end{array}$ & 9 & 38 & & & & \\
\hline $\begin{array}{l}\text { Combination } \\
\text { reduction } \\
<114 \%\end{array}$ & 9 & 8 & $<0.004$ & $53 \%$ & $83 \%$ & $75 \%$ \\
\hline
\end{tabular}




\begin{tabular}{|c|c|c|c|c|c|c|}
\hline $\begin{array}{l}\text { Combination } \\
\text { reduction } \geq \\
114 \%\end{array}$ & 8 & 39 & & & & \\
\hline $\begin{array}{l}\text { MRI >30\% } \\
\text { reduction in } \\
\text { diameter }\end{array}$ & 8 & 9 & 0.03 & $50 \%$ & $79 \%$ & $71 \%$ \\
\hline $\begin{array}{l}\text { MRI<30\% } \\
\text { reduction } \\
\text { diameter }\end{array}$ & 8 & 34 & & & & \\
\hline
\end{tabular}

Table 1

Associations between changes in US, SWE and MRI parameters at interim scanning and PCR at the end of NACT

\section{References}

1.Marinovich ML, Macaskill P, Irwig L, Sardanelli F, Mamounas E, et al. Agreement between MRI and pathologic breast tumor size after neoadjuvant chemotherapy, and comparison with alternative tests: individual patient data meta-analysis. BMC Cancer. 2015 Oct 8;15:662 2.Shin HJ, Kim HH, Ahn JH, Kim SB, Jung KH, Gong G, Son BH, Ahn SH. Comparison of mammography, sonography, MRI and clinical examination in patients with locally advanced or inflammatory breast cancer who underwent neoadjuvant chemotherapy. Br J Radiol. 2011 Jul;84:612-20 
3.Galbán CJ, Ma B, Malyarenko D, Pickles MD, Heist K, et al. Multi-site clinical evaluation of DWMRI as a treatment response metric for breast cancer patients undergoing neoadjuvant chemotherapy. PLoS One. 2015 Mar 27;10(3):e0122151. doi: 10.1371/journal.pone.0122151. eCollection 2015.

4.Bufi E, Belli P, Di Matteo M, Terribile D, Franceschini G, et al. Effect of breast cancer phenotype on diagnostic performance of MRI in the prediction to response to neoadjuvant treatment. Eur J Radiol. 2014 Sep;83(9):1631-8

5.Parikh J, Selmi M, Charles-Edwards G, Glendenning J, Ganeshan B, et al. Changes in primary breast cancer heterogeneity may augment midtreatment MR imaging assessment of response to neoadjuvant chemotherapy. Radiology. 2014 Jul;272(1):100-12.

6. Hylton NM, Blume JD, Bernreuter WK, Pisano ED, Rosen MA, Morris EA Locally advanced breast cancer: MR imaging for prediction of response to neoadjuvant chemotherapy--results from ACRIN 6657/I-SPY TRIAL. Radiology. 2012 Jun;263(3):663-72 7.Evans $\mathrm{A}$, Whelehan $\mathrm{P}$, Thomson $\mathrm{K}$ et al Differentiating benign from malignant solid breast masses: value of shear wave elastography according to lesion stiff-ness combined with gray scale ultrasound according to BI-RADS classification. Br J Cancer 2012 107:224-229

8.Berg WA, Cosgrove DO, Dore CJ, et al. Shear-wave elastography improves the specificity of breast US: The BE1 multinational study of 939 masses. Radiology 2012; 262:435-449.

9.Evans A, Rauchhaus P, Whelehan P, Thomson K, Purdie CA, Jordan LB, Michie CO, Thompson AM, Vinnicombe S. Does shear wave ultrasound independently predict axillary lymph node metastasis in women with invasive breast cancer? Breast cancer research and treatment. $2014 ; 143: 153-7$ 
10.Evans A, Armstrong S, Whelehan P, Thomson K, Rachhaus P, Purdie C, Jordan L, Jones

L,Thompson A, Vinnicombe S. Can Shear Wave Elastography Predict Response to Neo-adjuvant Chemotherapy in Women with Invasive Breast Cancer? BJC 2013 ;109: 2798-2802.

11.Lee SH, Chang JM, Han W, Moon HG, Koo HR, et al. Shear-Wave Elastography for the Detection of Residual Breast Cancer After Neoadjuvant Chemotherapy. Ann Surg Oncol. 2015 Dec;22 Suppl 3:S376-84.

12. Ma Y, Zhang S, Li J, Li J, Kang Y, Ren W. Comparison of strain and shear-wave ultrasounic elastography in predicting the pathological response to neoadjuvant chemotherapy in breast cancers. Eur Radiol. 2016 (epub head of print)

13. Jing H, Cheng W, Li ZY, Ying L, Wang QC, Wu T, Tian JW. Early Evaluation of Relative Changes in Tumor Stiffness by Shear Wave Elastography Predicts the Response to Neoadjuvant Chemotherapy in Patients With Breast Cancer. J Ultrasound Med 2016 Aug;35(8):1619-27. doi: 10.7863/ultra.15.08052. Epub 2016 Jun 14.

14.Evans A, Whelehan P, Thomson K, McLean D, Brauer K, Purdie C, Jordan L, Baker L, Thompson A.Quantitative shear wave ultrasound elastography: initial experience in solid breast masses. Breast Cancer Research 2010;12:R104.

15.Gounaris I, Provenzano E, Vallier AL, Hiller L, Iddawela M, et al. Accuracy of unidimensional and volumetric ultrasound measurements in predicting good pathological response to neoadjuvant chemotherapy in breast cancer patients. Breast Cancer Research and treatment 2011. Jun;127:459-69

16.Cosgrove DO, Berg WA, Dore CJ, et al. Shear wave elastography for breast masses is highly reproducible. European Radiology 2012; 22:1023-1032

17.Paluch-Shimon S, Friedman E, Berger R, Papa M, Dadiani M, Friedman N, et al. Neo-adjuvant doxorubicin and cyclophosphamide followed by paclitaxel in triple-negative breast cancer 
among BRCA1 mutation carriers and non-carriers. Breast Cancer Res Treat. 2016

May;157(1):157-65

18. Heil J, Kümmel S, Schaefgen B, Paepke S, Thomssen C, et al. Diagnosis of pathological complete response to neoadjuvant chemotherapy in breast cancer by minimal invasive biopsy techniques.Brit J Cancer. $2015 ; 113: 1565-70$

19. Avril S, Muzic RF Jr, Plecha D, Traughber BJ, Vinayak S, Avril N. 18F-FDG PET/CT for Monitoring of Treatment Response in Breast Cancer. J Nucl Med. 2016 Feb;57 Suppl 1:34S-9S 20.Patten DK, Zacharioudakis KE, Chauhan H, Cleator SJ, Hadjiminas DJ. Sentinel lymph node biopsy after neo-adjuvant chemotherapy in patients with breast cancer: Are the current false negative rates acceptable? Breast. 2015 Aug;24(4):318-20

21. Evans A, Armstrong S, Whelehan P, Thomson K, Rachhaus P, Purdie C, Jordan L, Jones L,Thompson A, Vinnicombe S. Can Shear Wave Elastography Predict Response to Neo-adjuvant Chemotherapy in Women with Invasive Breast Cancer? BJC 2013 ;109: 2798-2802. 